Article

\title{
Enhanced Algorithms for Estimating Tree Trunk Diameter Using 2D Laser Scanner
}

\author{
Ola Ringdahl ${ }^{1, *}$, Peter Hohnloser ${ }^{1}$, Thomas Hellström ${ }^{1}$, Johan Holmgren ${ }^{2}$ and Ola Lindroos ${ }^{3}$ \\ ${ }^{1}$ Department of Computing Science, Umeå University, SE-901 87 Umeå, Sweden; \\ E-Mail: peterh@cs.umu.se (P.H.); thomash@cs.umu.se (T.H.) \\ ${ }^{2}$ Department of Forest Resource Management, Swedish University of Agricultural Sciences, \\ SE-901 83 Umeå, Sweden; E-Mail: Johan.Holmgren@ slu.se \\ ${ }^{3}$ Department of Forest Biomaterials and Technology, Swedish University of Agricultural Sciences, \\ SE-901 83 Umeå, Sweden; E-Mail: Ola.Lindroos@slu.se \\ * Author to whom correspondence should be addressed; E-Mail: ringdahl@cs.umu.se; \\ Tel.: +46-907-865-722; Fax: +46-907-869-995.
}

Received: 8 July 2013; in revised form: 9 September 2013 / Accepted: 25 September 2013 / Published: 8 October 2013

\begin{abstract}
Accurate vehicle localization in forest environments is still an unresolved problem. Global navigation satellite systems (GNSS) have well known limitations in dense forest, and have to be combined with for instance laser based SLAM algorithms to provide satisfying accuracy. Such algorithms typically require accurate detection of trees, and estimation of tree center locations in laser data. Both these operations depend on accurate estimations of tree trunk diameter. Diameter estimations are important also for several other forestry automation and remote sensing applications. This paper evaluates several existing algorithms for diameter estimation using 2D laser scanner data. Enhanced algorithms, compensating for beam width and using multiple scans, were also developed and evaluated. The best existing algorithms overestimated tree trunk diameter by $c a$. $40 \%$. Our enhanced algorithms, compensating for laser beam width, reduced this error to less than $12 \%$.
\end{abstract}

Keywords: beam width compensation; multiple laser scans; stem diameter; forestry; accuracy and quality; terrestrial laser scanning; mobile laser scanning 


\section{Introduction}

Accurate estimation of tree trunk diameter is important both for traditional remote sensing applications and for advanced automation solutions in forestry. The main focus for remote sensing in forestry has generally been on describing properties and locations of trees. Features of relevance for forest related products and the operations related to forest management have been prioritized. Predictions of tree trunk attributes for entire forest areas are valuable for optimization of wood supply and for planning forest operations [1]. The diameter of a tree trunk is a good predictor for many features of interest (e.g., stem volume) [2], and is also inherently related to the location of the tree's centerpoint. Thus, accurate estimation of tree diameter is highly desirable for any remote sensing method working at tree level.

Forest machines used for fully mechanized cut-to-length (CTL) harvesting are technically advanced, with for instance automatic mechanical measuring of stem diameters and lengths during harvest. This information is processed in real-time optimization algorithms to support value maximizing cutting of stems into logs. Moreover, the information is transferred from the machines and used in central systems for managing wood supply of forest industries. The trend is towards even more advanced operation through, for instance, automation of crane functions [3] and autonomous navigation [4-6]. Several proposed solutions involve sensors mounted on a forests machine to create local tree maps of the environment in real-time [5,7,8]. This is typically achieved with a 2D laser scanner combined with SLAM (simultaneous localization and mapping) algorithms [9-11]. The resulting maps may be used for autonomous navigation, including path planning [7], documentation, and also for various semi-autonomous functions. The machine may for example automatically position the harvester head and grip a tree that the operator selects by pointing on a screen [12]. To achieve high precision in the SLAM algorithms, the center of the tree must be accurately determined [13]. Since a laser scanner senses the outer boundary of the more or less circular tree trunk, the estimated position of the tree's centerpoint depends on the diameter estimation. The diameter is important also to decide if a laser point cluster should be regarded as a tree or not $[14,15]$. Hence, the more accurate diameter estimation, the more accurate local map.

Recent progress suggests new interesting possibilities to combine such automatically generated local maps with airborne laser scanning (ALS). Through ALS, high precision geographical information is available for large areas. Height models of tree crowns and ground are created from ALS data, and tree crown segmentation algorithms can be applied to produce global tree maps with complete coverage. Several variables related to crown shape and size, for example stem volume [16], can then be calculated. Combined maps may be used to improve localization of a forest machine using matching algorithms, where positions from a global navigation satellite system (GNSS) are used only as starting positions [17,18]. This provides an alternative or supplement to GNSS, which has a typical position accuracy of \pm 10 meters in forest environments [19-21]. Today, manual field inventories at georeferenced sample plots are still needed to establish the models used to predict stem attributes from ALS data [22]. In the future, local tree maps generated by harvesters during normal forest operations could make it possible to collect more reference data to improve predictions and obtain more detailed stem data [23]. In this way, the normal harvest would constitute a destructive sampling, from which inference for similar 
forest areas could be made. Thus, the continuous and large-scale forest operations make it possible to set up a system for large-scale practicing and enhancing of ALS based algorithms. To combine global and local maps, trees in the maps have to be matched. Tree trunk diameter estimates may provide valuable information for this task.

In this article, we focus on the use of terrestrial 2D laser scanners for estimation of tree diameter. Such scanners provide scan lines in a typically horizontal plane, and have been used to estimate diameter and also location of the centerpoint of trees [14,15]. Jutila et al. [14] estimated tree trunk diameters using the angle between the edges and the shortest distance to a laser point cluster. They found the commonly used circle fit method to be significantly less accurate. Commonly used 2D scanners have large beam divergence with a spot size of 10-20 centimeters at a distance of 10 meters. This beam width is one possible reason for the overestimation of stem diameters reported in the literature (see e.g., [14]).

Other studies report on the usage of terrestrial 3D laser scanners. The algorithms often require a high resolution 3D point cloud, and are based on a variety of techniques such as the Hough transform [24-27], fitting of circles or cylinders [27-32], voxel based systems [33,34] and surface detection techniques [35]. In addition to diameter and position, high level tree features can be estimated. However, 3D scanners are expensive and computing intense compared to 2D scanners. Several of the applications mentioned above require real-time sensing with the equipment permanently mounted on a moving forest machine. One of the major challenges is that the sensor must endure and perform in year-around operations in rough terrain. Also, the used harvester technology is already expensive and a low cost sensor system is required. Robust 2D scanners have previously been successfully used on forest machines $[5,7,8]$ and for navigation of robots [36]. In this study we therefore investigated the possibility of enhanced algorithms for tree trunk diameter estimation. Several existing algorithms were evaluated and extended to compensate for beam width. Also, the possibility to improve the algorithms by fusion of several consecutive scans was studied.

The objectives of this study were to (1) compare several existing algorithms with new algorithms compensating for beam width; (2) to evaluate how the errors depend on tree trunk diameter and distance to the scanner; and (3) to evaluate various methods for using multiple laser scans. The paper is organized as follows. Section 2 describes the experiments, and in Section 3 existing and new diameter estimation algorithms are described. In Section 4, an evaluation of all described algorithms is presented. Section 5 contains a discussion of the results, and Section 6 finalizes with conclusions of the study.

\section{Study Outline}

Three existing algorithms for tree trunk diameter estimation (DEA) using a 2D laser scanner were evaluated. To improve the algorithms, we developed and evaluated two methods compensating for laser beam width, and two methods using multiple scans. This resulted in fourteen combinations in total. For the experiment, a SICK LMS 221 laser scanner was used. The angular resolution of the laser scanner was $0.25^{\circ}$, the field of view $100^{\circ}$, and the measurement range $80 \mathrm{~m}$. Each laser beam had a width of $0.6^{\circ}$ (ca. $14 \mathrm{~cm}$ at $10 \mathrm{~m}$ range).

Nine tree trunk sections with diameters in the range of 6-50 cm were used for the experiment (Table 1, Figure 1). The tree sections were placed one at a time in an indoor corridor, at distances varying between 
5 and $20 \mathrm{~m}$, with varying sides facing the laser scanner. In total, we measured 172 combinations of stem section diameters and distances, which from here on will be considered as independent observations of trees. For each measurement, the tree's real diameter was manually measured with mm-accuracy using a caliper at the spot where the laser beams hit. The location of the spot was determined by a Laserliner RangeXtender 30 line-laser detector.

Table 1. Tree species and diameter range for the nine tree trunk sections used in the experiment.

\begin{tabular}{lc}
\hline Species & Diameter Range (cm) \\
\hline Goat willow (Salix caprea) & $42.2-49.6$ \\
European aspen (Populus tremula) & $7.6-8.5$ \\
European aspen (Populus tremula) & $17.2-19.2$ \\
Scots pine (Pinus sylvestris) & $5.6-6.0$ \\
Scots pine (Pinus sylvestris) & $11.5-13.4$ \\
Scots pine (Pinus sylvestris) & $17.6-20.8$ \\
Silver birch (Betula pubescens) & $16.5-18.3$ \\
Silver birch (Betula pubescens) & $22.9-28.0$ \\
Silver birch (Betula pubescens) & $31.5-34.4$ \\
\hline
\end{tabular}

Figure 1. The nine tree trunk sections that were used in the experiment.

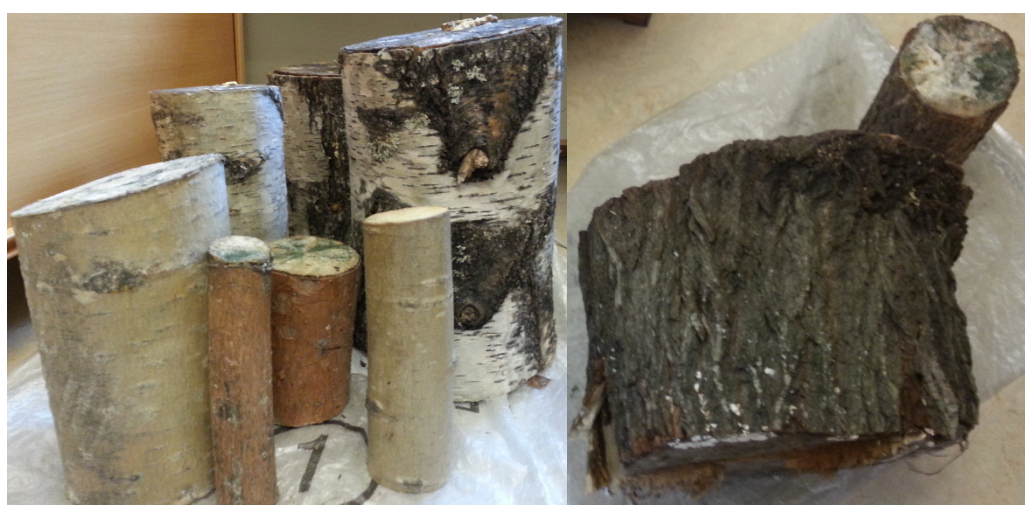

To identify trees in the laser scans, clusters of laser points fulfilling the following condition were extracted [14]:

$$
\left\|r_{i}-r_{i-1}\right\|<\Delta R_{\max }
$$

where $r_{i}$ is the range for the $i$ :th laser beam and $\Delta R_{\max }$ is a fixed threshold. $\Delta R_{\max }=0.5 \mathrm{~m}$ was used in this study. Tree clusters with less than three points were not used in the subsequent diameter estimation. Two samples were rejected by this restriction, resulting in 170 usable clusters. To filter out the walls of the corridor, only clusters having at least one point located within a $2.5^{\circ}$ wide sector centered at the middle laser beam were considered. In Jutila et al. [14], a more elaborated validation algorithm was applied to remove any cluster not fulfilling a number of defined criteria for a tree. In our case, no such validation was necessary since all samples were known to contain exactly one tree. 
Once the clusters were identified for the 170 samples, the algorithms for tree trunk diameter estimation were applied to the identified clusters. In the following section, the evaluated existing algorithms, and suggested improvements are described in detail. All algorithms use a cluster consisting of $n$ polar coordinates $\left(\left(r_{1}, \theta_{1}\right), \ldots,\left(r_{n}, \theta_{n}\right)\right)$ as input.

\section{Tree Trunk Diameter Estimation}

Three existing diameter estimation algorithms are evaluated (CF, TD, and VA). A suggested compensation for beam width resulted in an additional four algorithms (CFAA, CFEA, TDEA, and VAEA). Furthermore, all seven algorithms were used with multiple scans in two ways (MR and MD), giving a total of 14 algorithms that were evaluated. In the remainder of this section, existing algorithms and suggested improvements are described in detail.

\subsection{Existing Diameter Estimation Algorithms (DEA)}

Two triangle diameter estimation (TD) [36] uses the number of cluster points $n$, and the range $r_{m}$ to the center point (in the original work the shortest range was used) to calculate tree trunk diameter $d$ (see Figure 2). $\Delta \beta$ denotes the angular resolution of the laser scanner (in our case $0.25^{\circ}$ ):

$$
d=2 r_{m} \frac{\sin (\Delta \theta / 2)}{1-\sin (\Delta \theta / 2)}
$$

where $\Delta \theta=(n-1) \triangle \beta$.

Figure 2. Tree trunk diameter according to the TD algorithm using two right-angled triangles and the center point for the detected cluster.

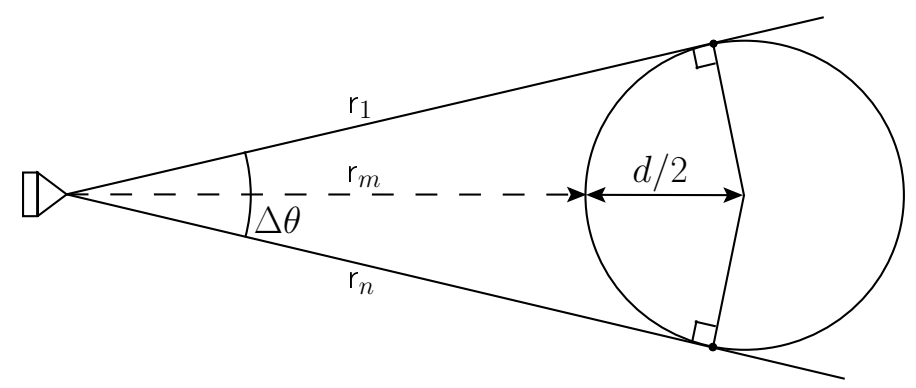

Diameter estimation based on viewing angle (VA) uses the first $\left(r_{1}\right)$ and the last $\left(r_{n}\right)$ cluster points, the number of cluster points $n$, and the resolution of the laser scanner $\Delta \beta$ to estimate trunk diameter $d$ [37] (see Figure 2):

$$
d=(n-1) \cdot \triangle \beta \cdot\left(r_{1}+r_{n}\right) / 2
$$

Circle fit $(C F)$ estimation, is based on fit of data in the detected cluster to the equation of a circle. Since the laser provides data in polar coordinates $(r, \theta)$, conversion to Cartesian coordinates $(x, y)$ is necessary: $x=r \cdot \cos (\theta), y=r \cdot \sin (\theta)$. We use an algorithm that fits the data to the equation $\left(x-x_{c}\right)^{2}+\left(y-y_{c}\right)^{2}=R^{2}$ by restating the problem as a linear least square problem. In this way, 
radius $R$ and center point $\left(x_{c}, y_{c}\right)$ can be computed both fast and accurately [38]. For the computations, a Matlab implementation by Izhak Bucher was used [39].

\subsection{Compensation for Beam Width (BWC)}

For the laser scanner used in the experiments, the width of each laser beam is $0.6^{\circ}$. This means that the actual angle at which reflection occurs can be $\pm 0.3^{\circ}$ off the nominal angle. Due to the width of the beam, an object will always be covered by at least three beams, regardless of its size, as illustrated in Figure 3. Whether a beam is reflected and registered by the laser scanner or not depends on the specific scanner construction and external variables such as angle of incident, reflectivity of the object and how large part of the beam that hits the object. This means that fewer than three beam reflections may be registered for very small objects.

Figure 3. Schematic view of three $0.6^{\circ}$ wide laser beams separated by $0.25^{\circ}$. The outer edges of the beams are illustrated by dotted lines in red, blue, and green, with the center of each beam marked with a solid line. Regardless of size, an object will be covered by at least three beams, due to the width of the beams, as can be seen around the solid green line in the center.

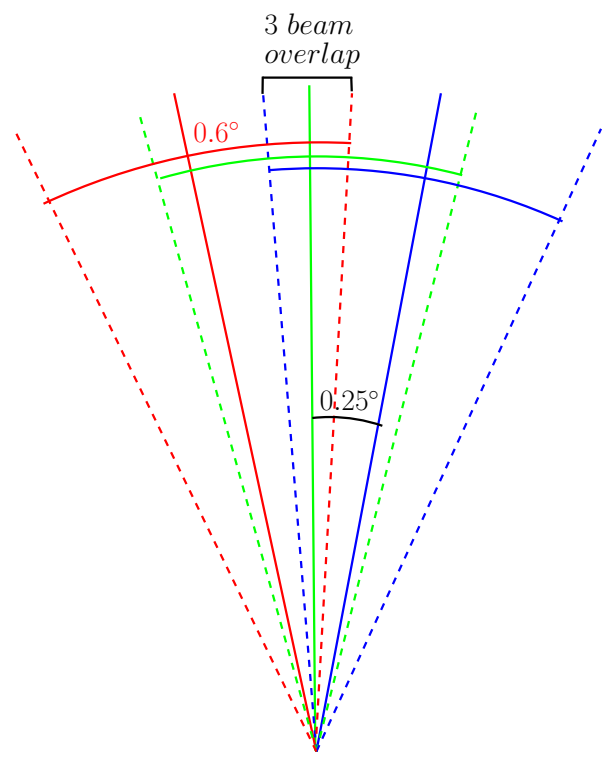

Since the beam is wider than the spacing between two beams, the center of the outermost beam will normally be outside the tree, leading to an overestimation of the diameter. To compensate for this, we developed enhanced versions of all algorithms, called Edge points Adjusted (EA). For the TD algorithm, the edge point adjustment is done as follows (algorithm TDEA) (compare with Equation (2)):

$$
d=2 r_{m} \frac{\sin ((\Delta \theta / 2)-\alpha)}{1-\sin ((\Delta \theta / 2)-\alpha)}
$$

For the VA algorithm, the edge point adjustment is computed as follows (algorithm VAEA) (compare with Equation (3)):

$$
d=((n-1) \triangle \beta-2 \alpha)\left(r_{1}+r_{n}\right) / 2
$$


For the $\mathrm{CF}$ algorithm, the edge point adjustment is done by moving the two outermost points towards the center of the cluster by an angle $\alpha$ (algorithm CFEA).

We also developed a second beam-width correction method for CF, called All points Adjusted (CFAA) where all points, not only the edge points, were moved towards the center by the angle $\alpha$ according to the algorithm:

if $\theta_{i}<\theta_{m}$ then $\theta_{i}:=\theta_{i}+\alpha$

else if $\theta_{i}>\theta_{m}$ then $\theta_{i}:=\theta_{i}-\alpha$

where $\theta_{m}$ is the angle to the center of the tree cluster (compare with $r_{m}$ in Equation (4)). $\theta_{i}$ is the $i$ th angle of a tree cluster. To calculate the center point $\left(r_{m}, \theta_{m}\right)$, the following algorithm is used:

if $N$ is odd then $\left(r_{m}, \theta_{m}\right)=(r, \theta)$ for the middle beam

else $\left(r_{m}, \theta_{m}\right)=$ mean of $(r, \theta)$ for the two middle beams

Which $\alpha$ to use was determined by an optimization procedure described in Section 4.

\subsection{Multiple Scans (MS)}

Since a laser scanner generates several scans per second (typically with a frequency of around $20 \mathrm{~Hz}$ ), it is possible to combine multiple scans even for mobile applications. All algorithms have been evaluated for two ways of using multiple scans (MS):

- Mean Ranges (MR): For each laser beam angle, compute and use the mean range of $N$ scans. The motivation is that the procedure will reduce noise and errors and the effect of outliers in the range readings, and possibly lead to better diameter estimates.

- Mean Diameter (MD): Calculate diameter estimations for each one of the $N$ scans, and report the mean value of all estimations. The assumption is that the scans are noisy and independent such that the mean of several diameter estimations is better than single estimates.

Unrealistic diameter estimates were not included in the performance evaluation as follows: For Mean Diameter (MD) - if a scan resulted in a diameter estimate $>100 \mathrm{~cm}$, the next consecutive scan was used to get the required number of scans. For Mean Ranges (MR) - if the mean of multiple ranges resulted in a diameter estimate $>100 \mathrm{~cm}$, the tree giving an erroneous reading was excluded from the evaluation. The only algorithms resulting in such large errors were the three based on circle fitting (CF, CFEA, and CFAA). In a real-time application, a validation algorithm could have filtered out clusters giving such large errors as part of a tree identification algorithm [37,40].

To summarize, we have three base algorithms (CF, TD, VA). Each one of these can be combined with beam width compensation (CFAA, CFEA, TDEA, VAEA). Finally these seven algorithms can be combined with the two different methods for fusing several scans (MR and MD), leading to a total of 14 algorithms that are evaluated (see Figure 4). Table 2 contains the abbreviations used. 
Figure 4. Mean absolute percentage estimation error and standard deviation as a function of number of scans for 14 algorithms. The legend shows the order of the algorithms for 10 scans, since this is used in the analysis of the results. (a) Mean estimation error; (b) Standard deviation.

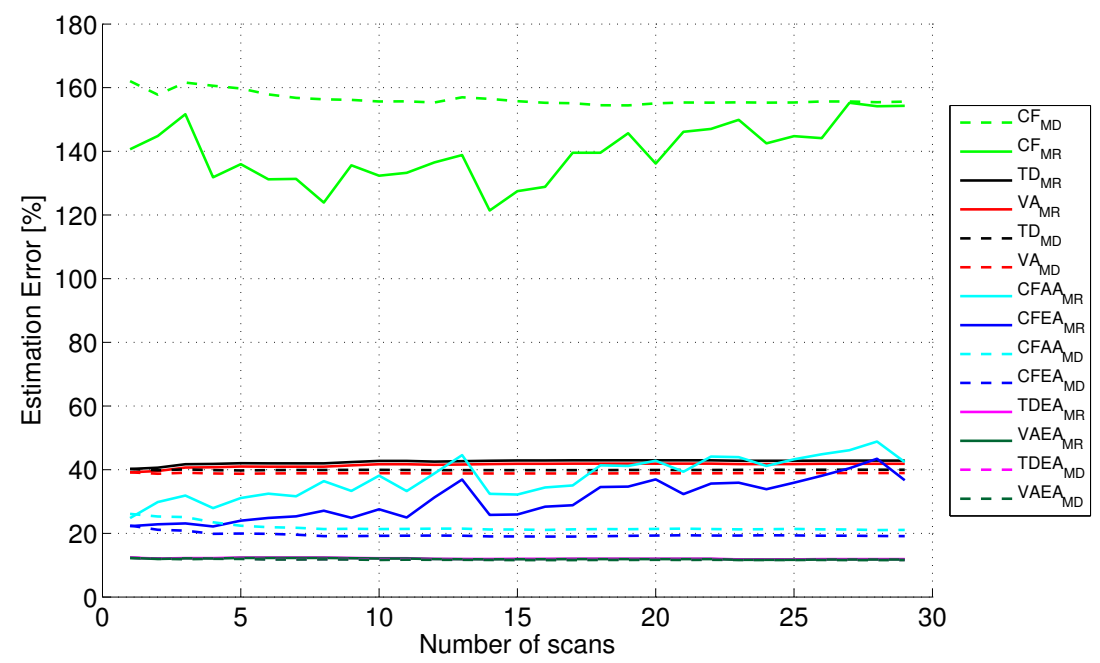

(a)

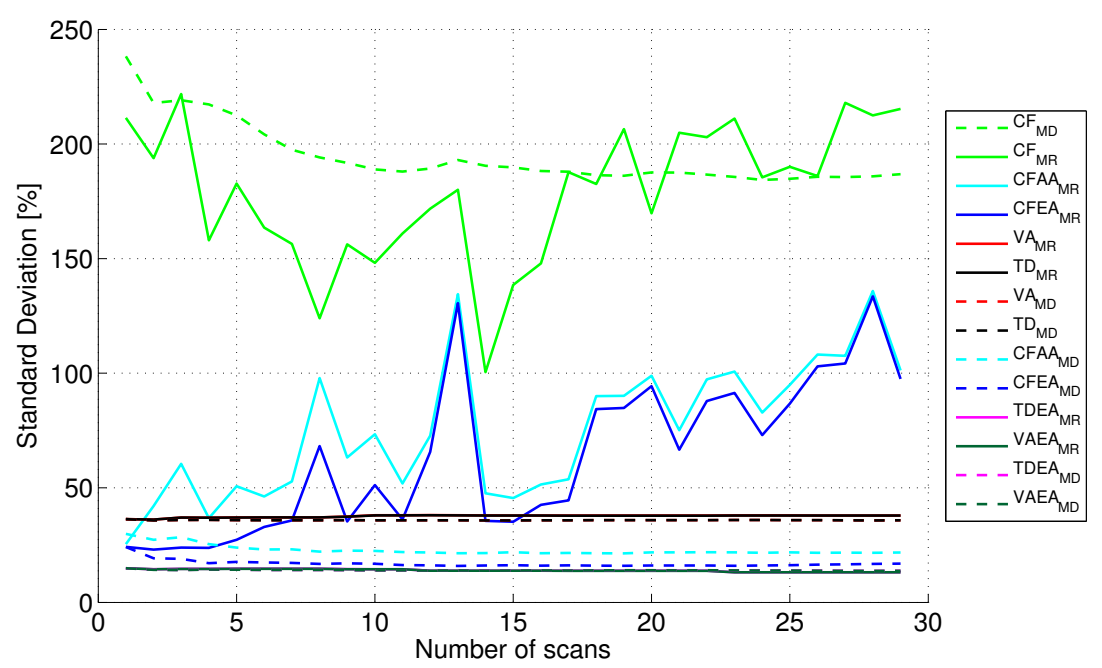

(b)

Table 2. Abbreviations.

\begin{aligned} & \hline DEA: Diameter estimation algorithms \\ & VA: Diameter estimation based on viewing angle \\ & TD: Two triangle diameter estimation \\ & CF: Circle fit \\ & BWC: Beam width compensation \\ & EA: Edge points adjusted \\ & AA: All points adjusted \\ & MS: Multiple scans \\ & MR: Mean ranges \\ & MD: Mean diameter \\ & \hline\end{aligned}




\subsection{Statistical Analysis}

The error values were evaluated for systematic bias (over- or underestimations) by using 1-sample T-tests to analyze if the mean value was significantly separated from zero within treatments.

Diameter estimation accuracy was evaluated as absolute error and as absolute percentage error. Absolute error was calculated as the absolute value of the difference between estimated and observed diameter. Absolute percentage error was calculated as the absolute error divided by the observed diameter and then multiplied by 100 .

To evaluate the effect of beam width compensation and multiple scans, the data was analyzed by use of analysis of covariance (ANCOVA). The full factorial model contained three fixed factors: diameter estimation algorithm (DEA; CF, TD \& VA), Beam width compensation (BWC; Yes \& No), and Multiple scans method (MS; MD \& MR). Thus, this ANCOVA model evaluated the resulting combinations of the three factors' levels (i.e., 12 algorithms). Tree trunk diameter and its distance from the scanner were entered as co-variates. In the analysis, the ANCOVA model was simplified by removing insignificant factors if it did not have any significant main or interaction effect.

Additionally, a separate two-way ANCOVA model was used for analyzing the additional beam width compensation method used uniquely for the $\mathrm{CF}$ algorithm (AA). Thus, within $\mathrm{CF}$, a full factorial model with beam width compensation (EA, AA \& No) and multiple scans method (MD \& MR) was used (i.e., six CF-based algorithms), with tree trunk diameter and distance from the scanner as co-variates.

A general linear model (GLM) was used for analyzing the ANOVA models (Minitab 16, Minitab Inc., State College, PA, USA). When needed, the dependent variable data were transformed to natural logarithms (Ln) to meet the the statistical tests' assumptions of normality and homoscedasticity. During the GLM procedure, pairwise differences were analyzed with Tukey's simultaneous test of means. When significant effects from covariates in ANCOVA analyses were found, the relationships were established by use of linear regression analysis. The critical level of significance was set to $5 \%$.

\section{Results}

The 14 algorithms described in Section 3 were tested and evaluated on clusters representing 170 trees. The number of points in each cluster (i.e., number of beams that hit a tree) varied from 3 to 20, with an average of 7.8 .

\subsection{Optimization of Beam width Correction and Multiple Scans Parameters}

The optimal beam-width correction angle $\alpha$ was determined by computing estimation errors with $\alpha$ ranging from $0^{\circ}$ to $0.4^{\circ}$ with $0.0029^{\circ}$ step $\left(5 \cdot 10^{-5}\right.$ radians), and choosing the $\alpha$ giving the lowest median error over a subsample of trees (the median was chosen to avoid the effect of outliers). This optimization procedure was repeated for 20 random subsamples, each one containing 50\% of the 170 available trees, and the average optimal $\alpha$ was finally selected. The average optimal $\alpha$ for TDEA and VAEA was $0.15^{\circ}$, and $0.22^{\circ}$ for CFEA and CFAA (Figure 5). In the continued analysis, these values were used. The standard deviations over subsamples for the optimal $\alpha$ were approximately $0.01^{\circ}$ for TDEA and VAEA, and $0.02^{\circ}$ for CFEA and CFAA. This suggests that the optimal value is very independent of 
the subsampling, and that the same optimal values would be estimated if using a separate data set for optimization.

Figure 5. Absolute estimation error for varying beam width correction angle $\alpha$.

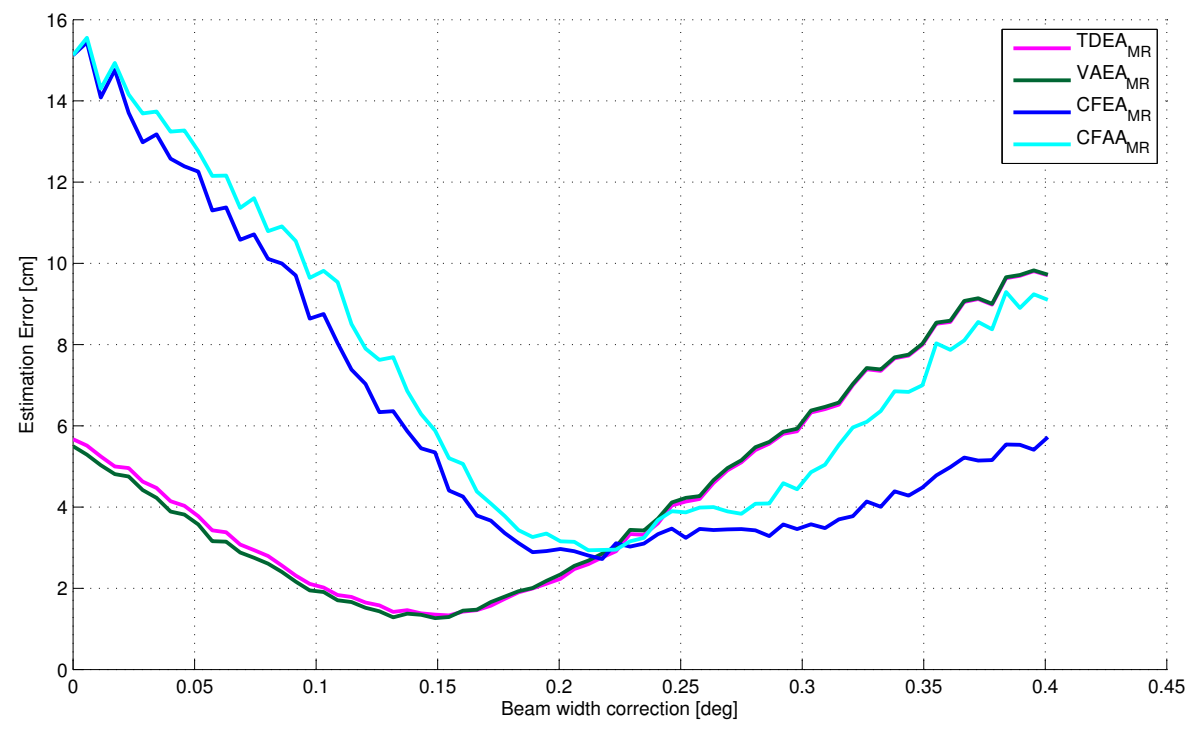

As described in Section 3.3, multiple scan were used in two ways. Combining diameter estimations from more than one scan with the MD method reduced both mean and standard deviation for the diameter estimation error (dotted lines in Figure 4). The effect was largest for the three circle fit algorithms. Combining several scans using the MR method (solid lines in Figure 4) did not improve performance in any significant way for any method. In the continued analysis, 10 scans were used for both MR and MD. This is a reasonable number to use in a real-time application, and using more scans did not significantly affect the error.

\subsection{Evaluation of Diameter Estimation Algorithms}

The absolute value of the Ln-transformed error in diameter estimation was significantly decreased $(p<0.001$ ) when using the beam width correction algorithm (BWC) edge point adjusted (EA) compared to not compensating, irrespective of diameter estimation algorithm (DEA) used (Table 3). Moreover, there was no significant difference in estimation error between the TD and VA algorithms. However, the CF DEA resulted in significantly higher errors than the other two DEA:s, although the difference decreased significantly when using the BWC method EA (significant interaction effect between DEA and BWC, $p<0.001$ ). There was no significant influence from the multiple scans (MS) methods or any of its related interaction effects $(p \geq 0.378)$, meaning that there was no difference in effect on the estimation errors between the two MS methods (MR and MD). The tree's real diameter and the distance to the tree significantly influenced on the absolute error $(p<0.001)$.

The absolute percentage error in diameter estimation displayed a response identical to the absolute error (Table 3); there were significant effects of DEA, BWC, the interaction between DEA and BWC, real diameter and the distance to the tree ( $p \leq 0.007)$, but no effect of MS or any of its related interaction effects $(p \geq 0.344)$. For these analysis, the ANCOVA models explained $60.2 \%$ of the observed variance 
( $R^{2}$-adj) in the absolute error data, and $55.8 \%$ in the absolute percentage error data. The main effect of BWC contributed with most ( $\mathrm{ca}$. 60\%-70\%) and the interaction between DEA and BWC contributed with least (slightly more than $1 \%$ ) of the model's explained variation.

Table 3. Mean estimation error and standard deviation (SD) for all tested combinations of diameter estimation algorithm (DEA) and beam width compensation (BWC) methods, using the MD multiple scan methods with 10 scans. Within columns, different superscripted lowercase letters indicate significant differences $(p<0.05)$ between the six combinations of algorithms and BWCs No and EA (two-way ANCOVA with Tukey test for Ln-transformed error values). Different superscript uppercase letters within columns indicate significant differences between BWCs within the CF algorithm $(p<0.05)$ (One-way ANOVA with Tukey test).

\begin{tabular}{|c|c|c|c|c|c|c|}
\hline \multirow{2}{*}{ DEA } & \multirow{2}{*}{ BWC } & \multirow{2}{*}{$\mathbf{n}$} & \multicolumn{2}{|c|}{ Absolute Error (cm) } & \multicolumn{2}{|c|}{ Absolute Percentage Error $(\%)$} \\
\hline & & & Mean & SD & Mean & SD \\
\hline \multirow{3}{*}{$\mathrm{CF}$} & No & 168 & $22.1^{a, A}$ & 14.8 & $155.6^{a, A}$ & 188.9 \\
\hline & EA & 170 & $3.7^{b, B}$ & 3.4 & $19.2^{b, B}$ & 16.9 \\
\hline & AA & 170 & $4.0^{B}$ & 4.3 & $21.3^{B}$ & 22.5 \\
\hline \multirow{2}{*}{ VA } & No & 170 & $5.8^{c}$ & 2.7 & $38.9^{c}$ & 35.8 \\
\hline & EA & 170 & $2.2^{d}$ & 3.0 & $11.7^{d}$ & 13.9 \\
\hline \multirow{2}{*}{$\mathrm{TD}$} & No & 170 & $6.0^{c}$ & 2.7 & $40.0^{c}$ & 35.8 \\
\hline & EA & 170 & $2.2^{d}$ & 3.0 & $11.8^{d}$ & 14.0 \\
\hline
\end{tabular}

For the CF algorithm, two different methods of BWC were tested, out of which the EA method provided smaller mean errors and standard deviations than the AA method (Table 3). However, the differences between the two methods were not significant when evaluating the three BWC alternatives with a two-way ANCOVA within CF for the Ln transformed absolute errors and absolute percentage errors $(p<0.001$ for the main effect of BWC in the ANCOVA, and $p<0.05$ for the pairwise Tukey test of AA and EA). However, both the EA and AA BWC methods significantly ( $p>0.05)$ improved the estimation errors compared to the estimation without any BWC. The ANCOVA also showed that the errors were significantly influenced by distance $(p<0.001)$ and by the real diameter when analyzing the absolute percentage error $(p<0.001)$ but not when analyzing the absolute error $(p=0.124)$. Irrespective of absolute error or absolute percentage error, there were no significant effect of MS methods or its interaction effects $(p \geq 0.094)$.

Since the multiple scans methods did not influence the errors, the following analysis are conducted on estimation with only one of the multiple scans methods (MD). Without angle correction, all algorithms systematically overestimated the diameter (Figure 6). When using angle correction, all estimations became more accurate. However, the TDEA and VAEA algorithms gave little variation in estimation errors, but a small systematic underestimation. Both angle correction methods of CF (CFEA and CFAA), on the other hand, resulted in large variation in error size but without systematic error. By using the minimum of 10 diameter estimations instead of mean in the MD method, the estimation error was reduced to $78 \%$ for $\mathrm{CF}$ and ca. $37 \%$ for VA and TD (compare with Table 3). For the four 
algorithms that compensates for beam width, the estimation error becomes larger if using min instead of mean. So compensating for beam width is still better than using min values on the original algorithms.

Figure 6. Distribution of diameter estimation errors (positive error values = overestimation, negative values $=$ underestimation) for the 7 algorithms, when applying the MD multiple scans method. $p$-values indicate the probability for obtaining the observed mean values when the true value is zero ( 1 -sample T-test). Thus, $p<0.05$ indicate systematic bias (over or under estimation). Zero error is indicated by dotted vertical line. $\mathrm{N}=170$, besides for $\mathrm{CF}$ for which $\mathrm{N}=168$.

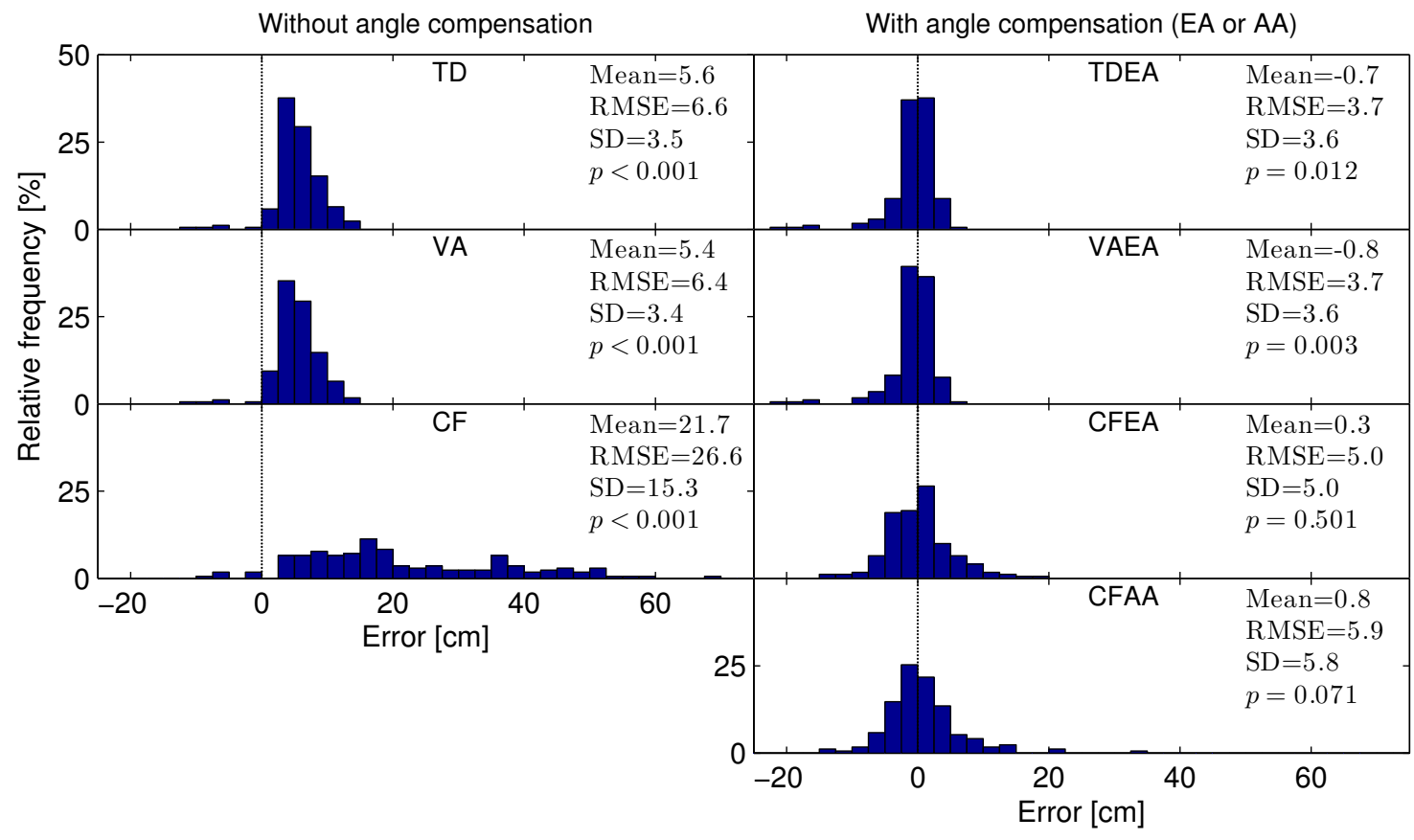

The ANCOVAs showed that the absolute percentage estimation error is influenced by tree trunk diameter. When analyzing the effects on one treatment combination (VAEA MD), it is shown that the absolute percentage error decreases with increasing tree trunk diameter and increases with increased distance (Figures 7 and 8). The relationship is statistically significant, but a simple regression analysis shows that the tree trunk diameter only explains $4 \%$ and $8 \%$ of the observed variation respectively ( $R^{2}$-adj; for untransformed and Ln-transformed absolute percentage error, respectively). Similarly, the distance to the tree as single predictor explains only $15 \%$ and $12 \%$, respectively, of the observed variation.

The experiment was not designed to evaluate the effect of tree species (bark texture), but allowed nevertheless for such an indicative analysis. Thus, for trees with diameters between 15 and $20 \mathrm{~cm}$ there were a reasonable (albeit unbalanced) number of observations for different species (95 birch, 213 aspen, and 72 pine). For this subsample, the tree species replaced the multiple scans factor in the general ANCOVA model. The analysis of the Ln transformed absolute errors and absolute percentage errors yielded basically the same results as previously: significant effects of DEA, BWC, the interaction between DEA and BWC, and distance to tree $(p<0.001)$. Moreover, it was indicated that tree species significantly affected the errors $(p<0.030$ ), with the efficiency of the BWC varying between tree species (interaction 
effect, $p=0.029)$. Thus, it was indicated a smaller estimation error for the birch bark $(p<0.05)$, than for the barks of aspen and pine (which did not significantly differ from each other $(p>0.05)$ ). Other effects and diameter did not significantly affect the errors in this subsample.

Figure 7. The estimation error decreases with increasing diameter $(n=170)$.

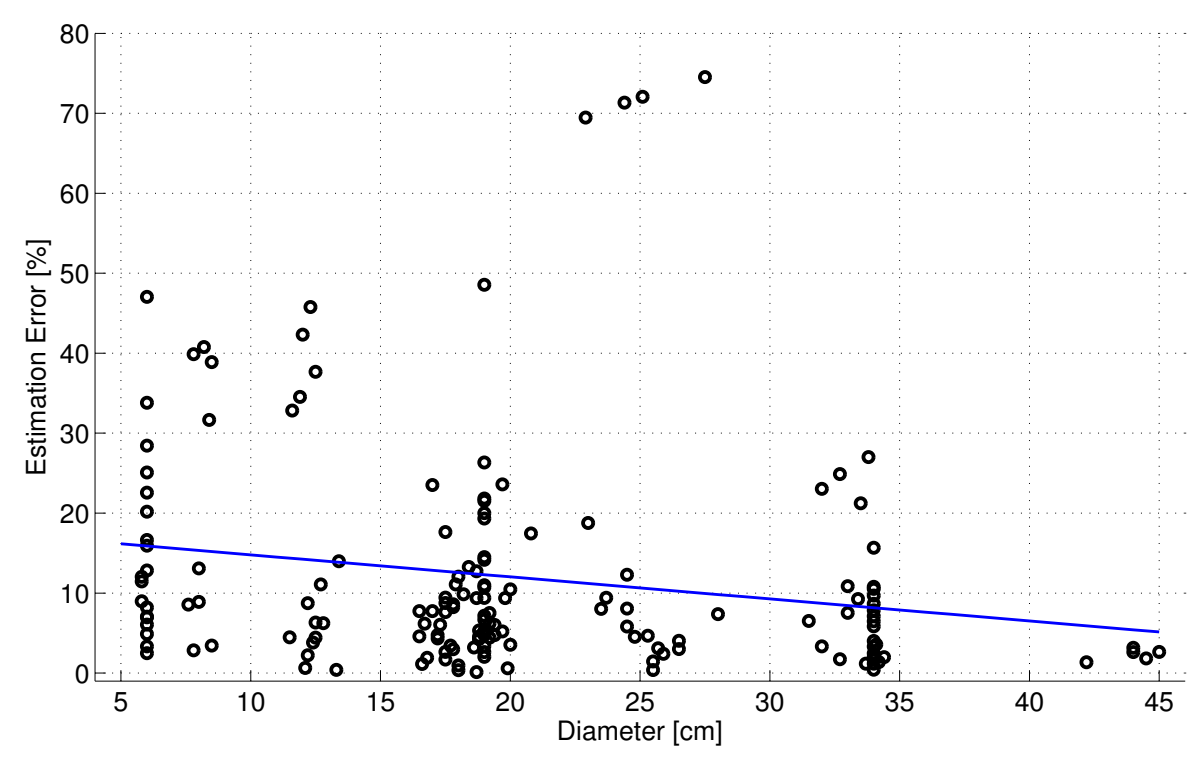

Figure 8. The estimation error increases with increasing distance. The size of the circles corresponds to the actual diameter of the trees $(n=170)$.

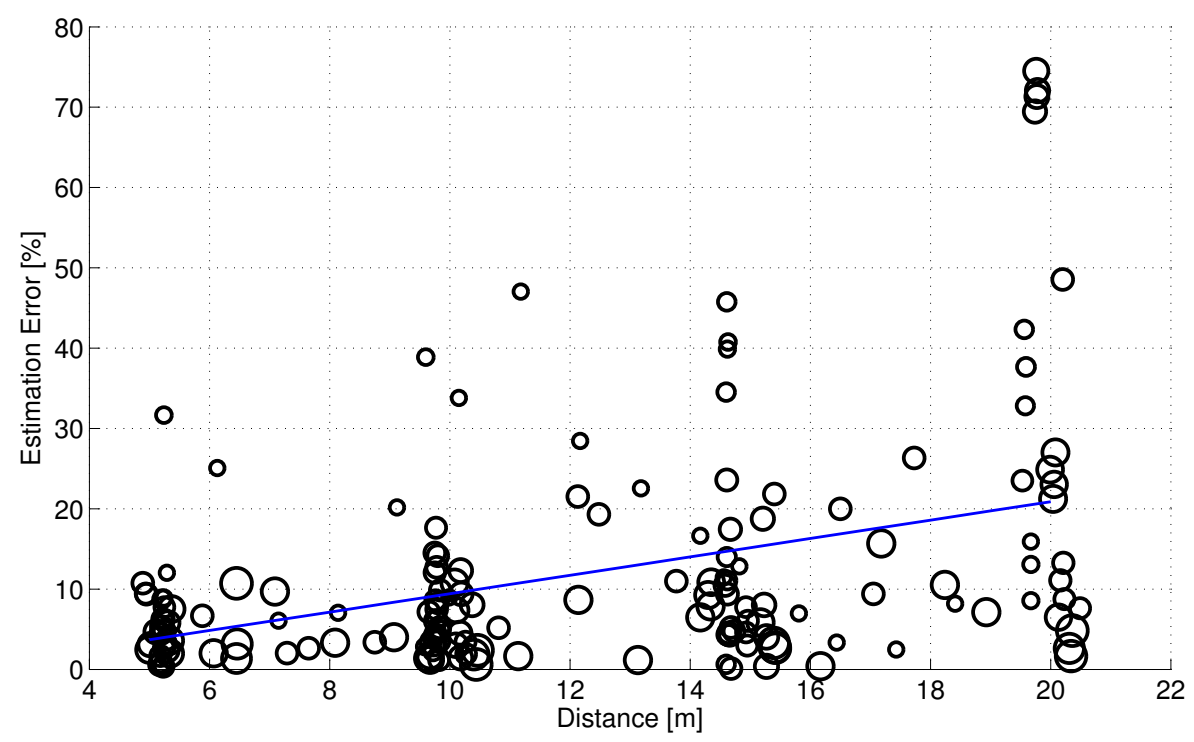

\section{Discussions}

The VA and TD methods gave very similar results with $39 \%$ and $40 \%$ error respectively. By compensating for beam width (TDEA, VAEA), the error was reduced to ca. 12\%. These two methods gave the best result in our study. The same effect was observed for all other methods as well; using beam width correction reduced the error by at least $70 \%$. The algorithm based on circle fitting (CF) did 
not perform well at all, giving $156 \%$ error on average. The algorithm was also very sensitive to noise in the data. If the points did not form well-behaved arcs, the diameter was overestimated significantly. CFEA and CFAA were a bit less sensitive than CF in this respect, as can be seen in Figure 6. The mean estimation error for all scans, including those giving diameter estimates $>100 \mathrm{~cm}$, was about $900 \%$ for the CF algorithm, while CFEA and CFAA gave around 300\% error. Removing all diameter estimates $>100 \mathrm{~cm}$ reduced the error to ca. $156 \%, 19 \%$, and $21 \%$ for CF, CFEA, and CFAA respectively. The major reason for CF performing so badly is probably the low number of cluster points. As previously mentioned, the number of beams that hit a tree (i.e the number of cluster points) varied from 3 to 20, with an average of 7.8. The TD algorithm without correction gave $11 \%$ error in a previous study [14] on 72 trees (filtered out from 277 found trees) with absolute mean and standard deviation $1.8 \mathrm{~cm}$ and $1.4 \mathrm{~cm}$ respectively. The corresponding results in our study were $40 \%, 6 \mathrm{~cm}$, and $2.7 \mathrm{~cm}$ (see Table 3 ). Jutila et al. [14] reported that the error increased with measuring range and were mostly positive for close ranges, indicating a systematic overestimation of diameter in close ranges. In Selkäinaho [37], the VA algorithm gave a relative diameter error between $0 \%$ and $58 \%$, measured on four trees with a diameter between 13 and $28 \mathrm{~cm}$. No average error was presented in that study. Zheng et al. [15] reported a maximum $9 \%$ error on 8 trees within 8 meters from the scanner using circle fitting. Without actual quantification, Jutila et al. [14] reported that a least squares circle fitting method gave "significantly larger" diameter errors than the the TD method (which gave $11 \%$ error in their study).

Our results show that diameter of and distance to the trees influenced the estimation errors. This makes perfect sense since the combination of diameter and distance is directly correlated to how many laser beams hit the tree, and hence to accuracy. The smaller the tree and the further away, the fewer laser beams hit the tree. At distances larger than $19 \mathrm{~m}$, the algorithms estimated approximately the same diameter $(\mathrm{ca} .7 \mathrm{~cm})$ for all trees with a real diameter less than $30 \mathrm{~cm}$. This gave large absolute percentage errors for trees with a real diameter around $30 \mathrm{~cm}$ and smaller errors for trees with a real diameter around $7 \mathrm{~cm}$, as seen in Figure 8. The reason for this is that, at this distance, these trees were hit by only 3 laser beams, giving the algorithms serious problems to differentiate between trees with different diameters. If a tree was hit by more than 3 beams, the diameter estimation improved, even at large distances.

All mentioned previous studies used substantially smaller datasets for evaluation, which may be one explanation to the different results compared to our study. Another plausible reason could be that the previous studies used a validation algorithm to filter out the best trees [14,15]. In our study, all trees regardless of the quality of the cluster were used, i.e., even if the points did not form well-behaved arcs, as long as the diameter estimates were less than $100 \mathrm{~cm}$. Furthermore, we measured trees at distances between 5 and 20 meters while previous studies measured trees between 2 and 10 meters, and a few up to $15 \mathrm{~m}[14,15,37]$. Since the error increases with distance, this could also explain the differences in results. Applying a previously developed validation algorithm [40], similar to the one used by Jutila et al. [14] and Zheng et al. [15], and only using trees closer than $15 \mathrm{~m}$ reduced the error for the TD algorithm from $40 \%$ to $22 \%$, TDEA from $12 \%$ to $6 \%$, CF from $156 \%$ to $59 \%$, and CFEA from $19 \%$ to $11 \%$ for a total of 41 trees (out of 170). Harder filtering reduces the error even more, but has not been investigated further since the purpose of this study primarily was to compare performance of several algorithms on all available data that beforehand is known to represent a tree. 
Estimation performance depends on the type of laser scanner. Previous studies [14,15,37] used a SICK LMS 291 with similar performance as the LMS 221 used in this study. Hence, the different results cannot be explained by the choice of laser scanner. However, using a scanner with less beam divergence could reduce the errors, especially for the methods not compensating for beam width.

When comparing real and estimated tree trunk diameter, it is crucial to know exactly where the laser beam hits the tree trunk, since tree trunks are well known for their taper [29] and for not having perfectly circular cross-sections [41]. In reported earlier work, no procedures to determine exact vertical position at which the laser hits the tree are mentioned. This raises questions about the accuracy of the reported error values since the natural variation in a tree's diameter is in the same order of magnitude as the average estimation error from the best algorithms in this study. Since our experiment was conducted in a controlled environment (indoors) we could fully focus on the diameter estimations. Moreover, the indoor experiment facilitated the work of determining where the laser beams hit the trees (using a laser light detector), which enabled recording of exact real diameter (using a caliper). Provided that tree stems can be detected, the improved algorithms presented in this paper will most likely work equally well outdoors. However, due to the generally noisy forest environment, tree detection is challenging and requires advanced filters to reduce influence of laser points coming from other objects than tree trunks (e.g., branches, stones and, bushes) [14,15,40].

\section{Conclusions}

We have evaluated several existing algorithms for estimating tree trunk diameter using a 2D laser scanner. The results show that algorithms based on circle fitting are sensitive to noise in the laser scans, and often give very large estimation errors. Two different algorithms based on viewing angle (TD and VA) give very similar result with around $40 \%$ error. Our suggested compensation for beam width results in a significant reduction down to $12 \%$ for the enhanced algorithms (TDEA and VAEA). In general, the observed errors for existing algorithms were higher in our study than in previously published research, but similar results were achieved when applying the same method to filter out data samples. A direct comparison is further complicated by the dependency on type of laser scanner, and on the amount and type of trees used for evaluation. Regarding influence of external factors, our study shows that the estimation error grows with increasing distance, in particular for trees with small diameter. In this study we considerably improved the tree trunk diameter estimation algorithms. Our enhanced algorithms may be used to improve SLAM generated local maps, which in combination with global ALS maps can be used for localization of forest machines. Improved diameter estimation is important also for several other forestry automation and remote sensing applications. Further efforts should focus on improved clustering and validation to extract laser points belonging to trees only, thereby improving the quality of data input to the estimation algorithms. Combining laser scanner with cameras is one possible approach that should be investigated towards these ends.

\section{Acknowledgments}

This work was partly funded by the European Commission (CROPS GA no 246252) and the Royal Swedish Academy of Agriculture and Forestry (KSLA; H11-0085-MEK, H11-0085-GBN). 


\section{Conflicts of Interest}

The authors declare no conflict of interest.

\section{References}

1. Holopainen, M.; Vastaranta, M.; Rasinmäki, J.; Kalliovirta, J.; Mäkinen, A.; Haapanen, R.; Melkas, T.; Yu, X.; Hyyppä, J. Uncertainty in timber assortment estimates predicted from forest inventory data. Eur. J. For. Res. 2010, 129, 1131-1142.

2. Zianis, D.; Muukkonen, P.; Makipaa, R.; Mencuccini, M. Biomass and stem volume equations for tree species in Europe. Silva Fenn. Monogr. 2005, 4, 1-63.

3. Shiriaev, A.; Freidovich, L.; Manchester, I.; Mettin, U.; La Hera, P.; Westerberg, S. Status of Smart Crane Lab Project: Modeling and Control for a Forwarder Crane; Technical Report; Department of Applied Physics and Electronics, Umeå University: Umeå, Sweden, 2008.

4. Hellström, T.; Ringdahl, O. Follow the past-A path tracking algorithm for autonomous vehicles. Int. J. Veh. Auton. Syst. 2006, 4, 216-224.

5. Hellström, T.; Lärkeryd, P.; Nordfjell, T.; Ringdahl, O.; Nordfjell, T. Autonomous forest vehicles: Historic, envisioned, and state-of-the-art. Int. J. For. Eng. 2009, 20, 31-38.

6. Ringdahl, O. Automation in Forestry-Development of Unmanned Forwarders. Ph.D. Thesis, Umeå University, Department of Computing Science: Umeå, Sweden, 2011.

7. Hellström, T.; Ringdahl, O. Real-time path planning using a simulator-in-the-loop. Int. J. Veh. Auton. Syst. 2009, 7, 56-72.

8. Öhman, M.; Miettinen, M.; Kannas, K.; Jutila, J.; Visala, A.; Forsman, P. Tree Measurement and Simultaneous Localization and Mapping System for Forest Harvesters. In Field and Service Robotics SE - 35; Laugier, C., Siegwart, R., Eds.; Springer: Berlin/Heidelberg, Germany, 2008; Volume 42, Springer Tracts in Advanced Robotics, pp. 369-378.

9. Wang, Z.; Huang, S.; Dissanayake, G. D-SLAM: Decoupled localization and mapping for autonomous robots. In Proceedings of the International Symposium of Robotics Research, ISRR 05, San Francisco, CA, USA, 12-15 October 2005; Volume 26, pp. 203-213.

10. Miettinen, M.; Ohman, M.; Visala, A.; Forsman, P. Simultaneous Localization and Mapping for Forest Harvesters. In Proceedings of the 2007 IEEE International Conference on Robotics and Automation, Roma, Italy, 10-14 April 2007; pp. 517-522.

11. Huang, S.; Wang, Z.; Dissanayake, G. Sparse local submap joining filter for building large-scale maps. IEEE Trans. Robot. 2008, 24, 1121-1130.

12. Mettin, U.; Westerberg, S.; Shiriaev, A.S.; La Hera, P.X. Analysis of human-operated motions and trajectory replanning for kinematically redundant manipulators. In Proceedings of the 2009 IEEE/RSJ International Conference on Intelligent Robots and Systems, St. Louis, MO, USA, 11-15 October 2009; pp. 795-800.

13. Dissanayake, M.W.M.G.; Newman, P.; Clark, S.; Durrant-Whyte, H.; Csorba, M. A solution to the simultaneous localization and map building (SLAM) problem. IEEE Trans. Robot. Autom. 2001, 17, 229-241. 
14. Jutila, J.; Kannas, K.; Visala, A. Tree Measurement in Forest by 2D Laser Scanning. In Proceedings of the 2007 IEEE International Symposium on Computational Intelligence in Robotics and Automation, Jacksonville, FL, USA, 20-23 June 2007; pp. 491-496.

15. Zheng, Y.; Liu, J.; Wang, D.; Yang, R. Laser scanning measurements on trees for logging harvesting operations. Sensors 2012, 12, 9273-9285.

16. Hyyppa, J.; Hyyppa, H.; Leckie, D.; Gougeon, F.; Yu, X.; Maltamo, M. Review of methods of small-footprint airborne laser scanning for extracting forest inventory data in boreal forests. Int. J. Remote Sens. 2008, 29, 1339-1366.

17. Rossmann, J.; Schluse, M.; Schlette, C.; Buecken, A.; Krahwinkler, P.; Emde, M. Realization of a Highly Accurate Mobile Robot System for Multi Purpose Precision Forestry Applications. In Proceedings of the 2009 International Conference on Advanced Robotics, ICAR 2009, Munich, Germany, 22-26 June 2009; pp. 1-6.

18. Rossmann, J.; Krahwinkler, P.; Schlette, C. Navigation of mobile robots in natural environments: Using sensor fusion in forestry. J. Syst. Cybern. Inform. 2010, 8, 67-71.

19. Andersen, H.E.; Clarkin, T.; Winterberger, K.; Strunk, J. An accuracy assessment of positions obtained using survey- and recreational-grade global positioning system receivers across a range of forest conditions within the Tanana Valley of interior Alaska. West. J. Appl. For. 2009, 24, 9.

20. Rodríguez-Pérez, J.R.; Álvarez, M.; Sanz-Ablanedo, E. Assessment of low-cost GPS receiver accuracy and precision in forest environments. J. Surv. Eng. 2007, 133, 159-167.

21. Naesset, E.; Jonmeister, T. Assessing point accuracy of DGPS under forest canopy before data acquisition, in the field and after postprocessing. Scand. J. For. Res. 2002, 17, 351-358.

22. Næsset, E.; Gobakken, T.; Holmgren, J.; Hyyppä, H.; Hyyppä, J.; Maltamo, M.; Nilsson, M.; Olsson, H.k.; Persson, A.s.; Söderman, U. Laser scanning of forest resources: The nordic experience. Scand. J. For. Res. 2004, 19, 482-499.

23. Holmgren, J.; Barth, A.; Larsson, H.; Olsson, H.k. Prediction of stem attributes by combining airborne laser scanning and measurements from harvesters. Silva Fenn. 2012, 46, 227-239.

24. Haala, N.; Reulke, R.; Thies, M.; Aschoff, T. Combination of Terrestrial Laser Scanning with High Resolution Panoramic Images for Investigations in Forest Applications and Tree Species Recognition. In Proceedings of the ISPRS Working Group V1 Panoramic Photogrammetery Workshop; Maas, H.G., Schneider, D., Eds.; Dresden, Germany, 2004; Volume 1, pp. 1-4.

25. Aschoff, T.; Spiecker, H. Algorithms for the automatic detection of trees in laser scanner data. Int. Arch. Photogramm. Remote Sens. Spat. Inf. Sci. 2004, 36, 71-75.

26. Aschoff, T.; Thies, M.; Spiecker, H. Describing forest stands using terrestrial laser-scanning. Int. Arch. Photogramm. Remote Sens. Spat. Inf. Sci. 2004, 35, 237-241.

27. Lindberg, E.; Holmgren, J.; Olofsson, K.; Olsson, H. Estimation of stem attributes using a combination of terrestrial and airborne laser scanning. Eur. J. For. Res. 2012, 131, 1917-1931.

28. Pfeifer, N.; Winterhalder, D. Modelling of tree cross sections from terrestrial laser scanning data with free-form curves. Int. Arch. Photogramm. Remote Sens. Spat. Inf. Sci. 2004, 36, W2.

29. Thies, M.; Pfeifer, N.; Winterhalder, D.; Gorte, B.G.H. Three-dimensional reconstruction of stems for assessment of taper, sweep and lean based on laser scanning of standing trees. Scand. J. For. Res. 2004, 19, 571-581. 
30. Forsman, P.; Halme, A. 3-D mapping of natural environments with trees by means of mobile perception. IEEE Trans. Robot. 2005, 21, 482-490.

31. Henning, J.G.; Radtke, P.J. Detailed stem measurements of standing trees from ground-based scanning lidar. For. Sci. 2006, 52, 67-80.

32. Moskal, L.M.; Zheng, G. Retrieving forest inventory variables with Terrestrial Laser Scanning (TLS) in urban heterogeneous forest. Remote Sens. 2011, 4, 1-20.

33. Gorte, B.; Pfeifer, N. Structuring laser-scanned trees using 3D mathematical morphology. Int. Arch. Photogramm. Remote Sens. 2004, 35, 929-933.

34. Hosoi, F.; Omasa, K. Voxel-based 3-D modeling of individual trees for estimating leaf area density using high-resolution portable scanning lidar. IEEE Trans. Geosci. Remote Sens. 2006, 44, 3610-3618.

35. Liang, X.; Litkey, P.; Hyyppä, J.; Kaartinen, H.; Vastaranta, M.; Holopainen, M. Automatic Stem Location Mapping Using TLS for Plot-Wise Forest Inventory. In Proceedings of the SilviLaser 2009, College Station, Texas, USA, 14-16 October 2009.

36. Bailey, T.; Nebot, E. Localisation in large-scale environments. Robot. Auton. Syst. 2001, 37, 261-281.

37. Selkäinaho, J. Adaptive Autonomous Navigation of Mobile Robots in Unknown Environments. Ph.D. Thesis, Helsinki University of Technology, Otaniemi, Finland, 2002.

38. Coope, I.D. Circle fitting by linear and nonlinear least squares. J. Optim. Theory Appl. 1993, 76, 381-388.

39. Mathworks. Circle fit; File Exchange, MATLAB Central. Available online: http://www.mathworks.com/matlabcentral/fileexchange/5557-circle-fit (accessed on 30 September 2013).

40. Hellström, T.; Hohnloser, P.; Ringdahl, O. Tree Diameter Estimation Using Laser Scanner; Technical Report, UMINF 12.20; Department of Computing Science, Umeå University: Umeå, Sweden, 2012.

41. Pulkkinen, M. On non-circularity of tree stem cross-sections: Effect of diameter selection on cross-section area estimation, Bitterlich sampling and stem volume estimation in Scots pine. Silva Fenn. 2012, 46, 747-986.

(c) 2013 by the authors; licensee MDPI, Basel, Switzerland. This article is an open access article distributed under the terms and conditions of the Creative Commons Attribution license (http://creativecommons.org/licenses/by/3.0/). 